\title{
GLOBALIZACIÓN Y DEPENDENCIA: LOS NUEVOS IMPASSES DEL DESARROLLO BRASILEÑO
}

\author{
Alexandre Comin*
}

\section{PRESENTACIÓN}

El desarrollo de Brasil en el siglo XX - y el de varios países latinoamericanos- es antes que nada la historia de una cambiante aunque recurrente tensión entre el esfuerzo por la industrialización y los límites impuestos al crecimiento económico por las restricciones externas. Con velocidad envidiable hasta los años 70 -un crecimiento del PIB del orden del 7\% anual- el tren del desarrollo descarriló en los años 80 y pasa actualmente por el más intenso proceso de reestructuración de toda su historia. Ahora en el contexto de la globalización económica, la industrialización pasa a estar amenazada por un nuevo conjunto de limitaciones económicas que tienen su causa última en el carácter subordinado de la inserción externa del país. El objetivo de este breve ensayo es apuntar los principales impasses y oportunidades que vive hoy el desarrollo brasileño, teniendo siempre como telón de fondo las contradicciones entre el enorme potencial de crecimiento y las limitaciones impuestas por las dificultades de la búsqueda de un mínimo de estabilidad macroeconómica. Por limitaciones de espacio el foco de atención estará centrado en el sector industrial así como a las políticas a él destinadas, relegando las conexiones con la política económica general -es decir, la macroeconomía- al mínimo indispensable para la comprensión del movimiento general. Por el mismo motivo, presto particular atención al análisis de la dimensión comercial de la política industrial, aquí referida como regulación del mercado interno, puesto que se presenta como decisiva para todo el periodo analizado, sobre todo en los años 90.

Al principio, se busca realizar una breve caracterización del patrón de industrialización en Brasil hasta los años 70. La sección siguiente atenderá a las principales causas del fracaso de este patrón, enfatizando la importancia del ascenso de la ideología neoliberal en el plano internacional. La sección IV discute los nuevos parámetros de la política industrial brasileña en los años 90 y sus contradictorios impactos sobre la estructura productiva. En la sección IV.1, son aducidos algunos comentarios sobre la importancia del Mercosur en este proceso. La sección V discute la continuidad de esta estrategia en la política industrial del actual Gobierno Fernando Henrique Cardoso, agravada todavía más por las peculiaridades de la estabilización y de la

Traducción del portugués: Ariel Jerez Novara

(*) Profesor de la Facultad de Economía y Administración de la Pontificia Universidad Católica de Sao Paulo (FEA/PUC/SP). Investigador del Centro Brasileiro de Analise y Planejamento (CEBRAP) y Doctorando del Instituto de Economía de la Universidad Estadual de Campinas (IE/Unicamp). fragilización externa puesta en marcha por este gobierno. La sección siguiente teje algunas observaciones respecto del nuevo Régimen Automotriz, enseñando que existe hoy en Brasil un ejemplo de política industrial que, debido a sus diferencias con la orientación general, apunta posibilidades mucho más auspiciosas que las prevalecientes en el conjunto de la industria. Por fin, en la sección VII, se intenta resumir los argumentos presentados y se subrayan los actuales impasses del desarrollo brasileño.

\section{LA FASE DE INDUSTRIALIZACIÓN POR SUSTITUCIÓN DE IMPORTACIONES}

Aunque el embrión de la industrialización brasileña pueda ser buscado incluso en el siglo XIX, se puede decir que sólo después de 1930 es cuando la industria pasa a ser la fuerza dinámica del desarrollo económico. Como resultado de la gran crisis mundial de esta década, el modelo anterior de crecimiento agroexportador entra en crisis y prácticamente impone la internalización de algunas actividades industriales, no ya como simple complemento de producción rural. Es por eso mismo que se puede hablar de política industrial en Brasil sólo a partir de este momento: es aquí cuando se observa una preocupación sistemática de la política económica con el desarrollo industrial'.

A partir de entonces y hasta la crisis de los años 80, el impulso básico del desarrollo brasileño residió en aquello que se acordó llamar la industrialización por sustitución de importaciones (ISI). En la definición de la principal autora sobre el tema, la ISI, en sentido lato, es "un proceso de desarrollo interno que tiene lugar y se orienta sobre el impulso de restricciones externas y se manifiesta, primordialmente, a través de una ampliación y diversificación de la capacidad productiva industrial"’. La dinámica económica que se instala en el país puede ser descrita como la secuencia de una serie de saltos industrializadores provocados por estrangulamientos externos que limitaron la capacidad de importar. Una vez sustituida parte de la pauta importadora debido a estas restricciones, la continuidad del crecimiento provocaba nuevos estrangulamientos y de nuevo el estímulo a la substitución de otros productos se hacía presente. Alcanzando

(1) SUZIGAN (1966:7), que sirve como introducción al estudio de la política industrial en el Brasil. Ver también el clásico estudio de BAER (1985)

(2) TAVARES (1972:41). Otro estudio clásico sobre el asunto es el de FURTADO (1982:cap.XXXII) 
al principio bienes de consumo no duradero y algunas materias primás básicas, la industria se fue diferenciando hasta abarcar los bienes de consumo durable y otros insumos intermedios, y terminó por afectar a la industria pesada y de bienes de capital y a algunos segmentos de alta tecnología.

Aunque esta dinámica encuentre sus nexos causales esencialmente en el ámbito de la economía, es decir, entre el estrangulamiento externo y la diversificación industrial, el papel del Estado fue fundamental para articular estos movimientos. Al controlar los flujos de bienes y capital entre la economía local y la exterior, cabe al gobierno federal una función reguladora de las restricciones externas, modelando la escasez de divisas con el objetivo de acelerar la inversión substitutiva: es decir, favoreciendo la importación de bienes de capital e insumos y poniendo dificultades a la entrada de productos acabados.

Sintéticamente, los instrumentos de promoción a la industria fueron, por un lado, la protección de la producción interna y, de otro, un conjunto de incentivos a la inversión y a la producción. Del lado de la protección, se destaca la protección tarifaria, y más importante todavía, las restricciones no tarifarias, como las exigencias de índices de nacionalización de productos, la prohibición de importación de mercancías con producción local y una serie de medidas administrativas adoptadas en diversos momentos para contener las importaciones consideradas indeseables ${ }^{3}$. Otro elemento importante de regulación fue el cambio, no tanto por su valor, que muchas veces estaba por encima de un nivel "de equilibrio", sino sobre todo por una serie de innovaciones en su gestión: tasas múltiples de pago (favoreciendo bienes de producción y dificultando bienes de consumo), presupuestos de cambio discrecional y medidas más fuertes de contención de las importaciones en los momentos más agudos de estrangulamiento. Todo esto hizo que la manipulación de las tasas cambiarias sirviesen a los diversos propósitos específicos de la industrialización.

Del lado del fomento, la lista de los instrumentos adoptados sería demasiado larga para este artículo. Cabe destacar las políticas de financiación y los incentivos fiscales. En el primer caso, la ausencia de finanzas industrializadoras, es decir, de una relación entre sistema financiero e industria que sirviese para trasvasar del primer sector las inversiones para el segundo, hizo que el gobierno federal crease sus propias agencias de financiación de la inversión para la producción industrial. Primero, al Banco do Brasil, y a partir de los años 50, al Banco Nacional de Desenvolvimiento Económico e Social (BNDES), les correspondieron las funciones de financiación de largo plazo que fueron fundamentales para adelantar los capitales necesarios para las crecientemente voluminosas inversiones, no sólo del sector privado nacional sino también del propio Estado.

Lo importante aquí es retener el hecho de que estas y otras muchas políticas e instrumentos tuvieron una orientación y resultados de naturaleza eminentemente vertical, es decir, sectorial. Desde el principio, la política industrial brasileña cumplió las principales funciones de cualquier política de desarrollo: crear las condiciones para la diversificación del parque productivo interno, haciendo avanzar la economía en los sectores de mayor complejidad técnica, integrando sectores y complejos industriales de manera tal como para producir, a finales de los 70, una de las más completas estructuras industriales del Tercer Mundo. La soberanía sobre el mercado interno por parte del Estado nacional y su consciente regulación con vistas a la internalización secuenciada de los sectores industriales es la marca distintiva de la ISI.

De este modo, los ritmos de la diversificación productiva sobre la que avanza el modelo sustitutivo no puede ser entendida sin una referencia a la regulación estatal, que lo direcciona y determina las proporciones que esta diferenciación fue asumiendo a lo largo del tiempo. Si en los 30 y 40 la elección de los sectores prioritarios (siderurgia, papel y celulosa) estuvo poco planificada, a partir de los 50, el targeting, es decir, la elección consciente de los sectores industriales y la coordinación de los diversos instrumentos en pro de los mismos, se convierte en regla del desarrollo brasileño. Cabe destacar dos momentos de este proceso.

En la segunda mitad de los años 50, durante el gobierno Juscelino Kubitschek, es implantado el Plan de Metas, que establece objetivos precisos, temporalmente definidos, de implantación de segmentos prioritarios de infraestructura económica y de los sectores industriales. Entre estos, la importancia mayor la tuvieron los sectores de bienes de consumo durable, con relieve de la industria automovilística. La novedad principal aquí, entre otras, fue un conjunto de instrumentos volcados en la atracción de inversiones extranjeras, que desde entonces pasan a liderar ramos enteros de la industria nacional, sobre todo en los segmentos más dinámicos y de mayor complejidad tecnológica ${ }^{4}$.

El segundo momento, ya durante el régimen militar implantado en 1964, es el Segundo Plan Nacional de Desarrollo (II PND), entre 1974 y 1979 . El más ambicioso de los planes de promoción industrial tenía como objetivo completar la estructura productiva en sus segmentos básicos -petroquímica y otros insumos intermedios, bienes de capital, etc.- pero también para sustraer a la internacionalización sectores de punta como, armamentos, aviación, energía nuclear, informática y telecomunicaciones.

Por fin, cabe llamar la atención hacia un hecho hasta aquí olvidado: la política industrial en Brasil, más allá de su actuación sobre el sector privado, nacional o extranjero, hace del Estado el agente central de la industrialización. Desde la histórica constitución de la Companhia Siderúrgica Nacional en 1941, pasando por los holdings estatales en sectores como el petróleo, la minería, electricidad, ferrocarriles, o los de telecomunicaciones, etc., y llegando a Nuclebras (energía nuclear) en los años 70, la industrialización de Brasil tuvo como presupuesto el desarrollo ininterrumpido de un poderoso sector productivo estatal (SPE). En éste cabían los segmentos que por motivos diversos -por elevadas inversiones, largos plazos de maduración, complejidad tecnológica, altos riesgos, etc.- no podían ser asumidos por el capitalismo privado nacional o todavía, debido a consideraciones estratégicas, no fue entregado al sector extranjero. Esto sumado a lo que ya fue dicho anteriormente sobre los bancos públicos implica reconocer que la formación de la SPE fue decisiva para el desarrollo del empresariado nacional y también para el flujo de capital extranjero.

Sin espacio para realizar un análisis más cuidadoso de las relaciones entre la política industrial y los condicionamientos 
macroeconómicos, cabe sólo añadir un comentario que será retomado más adelante: claro está que hubo diversos momentos en que los objetivos de la estabilización macroeconómica entraron en conflicto con los proyectos desarrollistas. En rigor, este conflicto es característico de cualquier economía capitalista, en la cual el crecimiento siempre corre el riesgo de provocar distorsiones en el nivel general de precios y/o en la balanza de pagos. Incapaz de eliminar este conflicto, la ISI tuvo la virtud de hacer del crecimiento una especie de "fuga hacia adelante", es decir, una solución temporal pero recurrente a los desequilibrios y conflictos generados por las sucesivas olas industrializadoras ${ }^{5}$. Las consecuencias de este modo particular de combatir los desequilibrios provocados por el crecimiento con más crecimiento fueron, en el plano interno, la inflación permanente como recurso necesario al financiamiento del Estado, y, en el externo, la utilización de capital extranjero para financiar la economía como un todo. Desde los años 50 dependiente de las inversiones directas de capital (empresas multinacionales) para impulsar la industrialización, Brasil pasa, en la segunda mitad de los años 60 , a depender cada vez mas del crédito externo privado (bancos internacionales). Como veremos a continuación, la crisis de la ISI será también la crisis de estos mecanismos básicos de "la huida hacia adelante".

\section{LA CRISIS DEL MODELO ISI}

Ya a finales de la década del 70 era posible observar las primeras señales de ruptura del patrón de industrialización basado en la ISI: en el campo económico, la desaceleración de las inversiones privadas y el relativo deterioro de las variables macroeconómicas; en el campo político, crecían las protestas de los excluidos del progreso económico contra la dictadura militar y hasta incluso la alta burguesía comienza a oponerse a la "excesiva estatización" de la economía brasileña. Pero es recién en la década de los 80 que la crisis del modelo, al mismo tiempo una crisis de modelo y del Estado que la hacía viable, se manifiesta claramente, sumergiendo la economía más dinámica del siglo hasta entonces en un largo periodo de estagnación e inestabilidad macroeconómica que será conocida como la Década Perdida. Al mismo tiempo, la dictadura militar pierde legitimidad y pone en marcha una lenta transición hacia un régimen civil (1985) y finalmente democrático en el ámbito de la presidencia de la República (1990).

Visto en perspectiva, puede decirse que forma parte de la propia lógica de la ISI que encuentre en algún momento dificultades crecientes para avanzar hasta el punto en que se vuelve incapaz de promover nuevas inversiones: se podría hablar entonces del agotamiento del modelo. En este sentido, el pasaje de la industria ligera, donde el proceso tuvo inicio, hacia la pesada (bienes de capital y sectores intensivos en tecnologías) trae problemas cada vez mayores. De un lado, la ampliación de la relación capital/producto, la elevación de los plazos y riesgos en los nuevos sectores señalan la problemas para las oportunidades de inversión privada, lo que sólo puede en parte ser compensado por el recurso a la estatización. De otro, la ambición de inter-

(5) La expresión es de FIORI (1986), que ha emprendido un amplio análisis de las características políticas del desarrollo brasileño. nalizar segmentos de la frontera de la acumulación capitalista conlleva una dificultad creciente para atraer los capitales externos, dado que, desde el punto de vista de éstos ya no se trata sólo de traer tecnologías sino, por el contrario, compartir recursos tecnológicos de vanguardia, los más prometedores en términos de plusvalía prospectiva ${ }^{6}$.

A esto puede agregarse otros problemas que, aunque no puedan ser considerados como intrínsecos a la ISI, ocurrieron de forma tan persistente en el caso brasileño, así como en los demás países latinoamericanos, que son parte integrante de los impasses que se observan en la actualidad. El más importante se refiere a la casi nula internacionalización de la capacidad de generar conocimientos, técnicas y productos nuevos. En este sentido, la industrialización atrasada de América Latina, estuvo mal capacitada para instrumentalizar, absolver y copiar la tecnología generada en los países del capitalismo central. Aunque haya sido capaz, en algunos casos de forma sorprendentemente rápida, de generar condiciones para la utilización de las técnicas modernas, la industria permaneció fuertemente dependiente de los influjos externos para hacer efectiva esta utilización, sobre todo para las actividades de las empresas extranjeras. Excepción hecha a algunas iniciativas gestadas en el núcleo del II PND, como la aviación y algunas tecnologías del sector de las telecomunicaciones, el potencial innovador es bastante limitado.

En otras palabras, el desarrollo fue capaz de internalizar nuevas formas de producir pero no las nuevas formas de competir, que presuponen esa capacidad innovadora, en términos de nuevos productos, nuevos procesos y nuevos mercados ${ }^{7}$. Con el pasaje de la Segunda a la Tercera Revolución Industrial, esta deficiencia, en sí misma bastante grave, se convierte en un obstáculo de primer orden en el nuevo contexto global que se pretende discutir más adelante.

El segundo problema se refiere al hecho de que la política industrial ha dado un énfasis escaso al objetivo de insertar al país en los flujos internacionales de comercio. Es como si el empeño por la sustitución de importaciones -y su corolario de ineficiencia y desfase tecnológico- robase recursos e intereses necesarios a un esfuerzo más significativo. Nuevamente con la excepción del II PND, y en parte sobre la presión de las crisis petroleras de los 70, poco fue hecho en el sentido de estimular empresas y sectores para disputar el mercado internacional. Cabe señalar que esta oposición no es un producto de los condicionantes económicos de la ISI: los ejemplos de algunos países asiáticos muestran que la protección al mercado interno, más fuerte incluso que la aplicada en América Latina, puede, en algún momento del tiempo, ser acompañado de políticas específicas de promoción de las exportaciones ${ }^{8}$. En otras palabras, la ISI en Brasil aceptó pasivamente el estrangulamiento externo como parte integrante de su desarrollo, sin buscar formas de romperlo de forma activa, impulsando el esfuerzo exportador dentro de sectores nuevos, al menos hasta los años 70 .

(6) Otras dificultades intrínsecas al modelo y que se manifiestan de forma acumulativa pueden ser mencionadas: la elevada concentración e ineficacia que se derivan de las bajas escalas de producción, en relación a los países centrales, la distorsión en la utilización de los factores de producción debido al uso de tecnología externa, que absorbe mucho capital y genera pocos empleos, etc. Estos y otros problemas son examinados por TAVARES (1974:53).

(7) Las expresiones aparecen en BATISTA (1993:220). Para un abordaje más amplio ver FANJZYL $\operatorname{BER}(1983: 171)$

(8) Para una discusión sobre este punto véase EVANS (1984), PINHEIRO (1987) y FANJZYLBER (1983:116). Añádase que hoy día, debido a la contraofensiva neoliberal en América Latina, algunos tigre asiáticos, sobre todo Corea, muestran niveles de protección, tanto comercial como para las inversiones asiáticos, sobre todo Corea, muestran niveles
extranjeras, mucho mayores que los de Brasil. 
Estas consideraciones, no obstante, no son suficientes para explicar la forma concreta cómo se produjo el fracaso de la ISI en los años 80. Es preciso hacer referencia, aunque de forma sumaria, a los condicionantes más amplios de la inserción dependiente de Brasil en el escenario internacional. Frente a las nuevas constricciones externas procedentes de la crisis mundial de los años 70, el país intentó mantener sus elevados índices de crecimiento económico recurriendo al mercado privado de capitales como forma de equilibrar su balanza de pagos. Este fenómeno nuevo -un amplio mercado privado de créditos internacionales fue decisivo para el desenlace de la crisis: por primera vez se hacía posible superar el estrangulamiento externo aumentando la capacidad de importar por medio del endeudamiento 9 .

Los funestos resultados de esta estrategia son bastantes conocidos; con la crisis mexicana de insolvencia en 1982, prácticamente toda América Latina entra en la peor fase de crisis de toda su historia: bajo la orientación estricta del Fondo Monetario Internacional (FMI), estas economías entran en una nueva fase de estagnación, desequilibrios externos, inflaciones galopantes y un severo deterioro de sus finanzas estatales ${ }^{10}$. Sin entrar en más detalles, cabe señalar que esta crisis fue simultáneamente una crisis de desarrollo y una crisis del Estado que le daba soporte. De este modo, el crecimiento volcado al mercado interno fue sustituido por el esfuerzo desesperado por la generación de divisas. Los recursos públicos volcados a la inversión -pública y privada- se agotaron rápidamente. La atracción de capitales externos, antes factible, se convirtió en un objetivo al mismo tiempo indispensable e inalcanzable.

Sin embargo, sería necesario más de una década para que se hiciese evidente esta doble dimensión de la crisis: aunque en los años 80 , vistos en retrospectiva, hayan significado la muerte de la política industrial, no se puede decir que los gobiernos hayan abdicado en su intención de mantener como mínimo algunas de las políticas del periodo anterior. En el caso de la industria informática, ejemplo aislado, se intentó inclusive una nueva forma de promover la internacionalización de la actividad industrial: la llamada reserva de mercado, que buscaba crear una industria de capital nacional para el sector, pero con resultados poco animadores, dado que, entre otros problemas, no fue capaz de absorber las nuevas formas de competir. Pero, para ser breve ${ }^{11}$, la crisis del Estado -fiscal, de regulación y de legitimidad - en medio de la crisis económica general simplemente impidió el mantenimiento de funciones básicas, en términos de inversión, financiación e inducción de crecimiento.

Más que una crisis, la situación internacional trajo a los países dependientes un nuevo contexto: al menos para América Latina, los países centrales, haciendo uso de su hegemonía sobre las agencias multilaterales, el FMI en primer lugar, pasaron a interferir de forma creciente en las políticas económicas de los países insolventes. De este modo, las presiones por el equilibrio fiscal, por el esfuerzo exportador y por la contención de la demanda interna (para disminuir importaciones) hicieron algo más que arrojar a estas economías al laberinto de la estagflación: inviabilizaron cualquier tentativa de mantener el patrón anterior de desarrollo.

(9) Para un análisis detallado, ver CRUZ (1984:28).

(10) CARNEIRO (1993) presenta un amplio panorama de los aspectos macroeconómicos de la crisis brasileña de los años 80 .

(11) Al respecto, ver ERBER y VERMULM (1993:38).
Si no bastase este cuadro macroeconómico, los países centrales, sobre todo los EUA, pasaron a usar su supremacía para interferir en otros asuntos internos de los países como Brasil, más directamente relacionados con la política industrial. La ascensión del neoliberalismo en los países anglosajones pasa cada vez más a dominar el escenario internacional y a someter a las élites de América Latina a un nuevo ideario económico y social. Las palabras de orden son apertura comercial y de flujos de capital, la privatización de empresas estatales y el desmantelamiento del aparato público de una forma general, y sus funciones reguladoras en particular.

Actuando directamente ${ }^{12}$, o a través de agencias como el FMI o el Banco Mundial, los EUA logran imponer una nueva agenda de política económica a la mayoría de los países de América Latina que fue conocida como el Consenso de Washington $^{13}$. En su esencia -la triada neoliberal- consiste en un movimiento en dirección a la apertura comercial, la desregulación y la privatización. Justamente la antítesis de aquello que convirtió el desarrollo posible: regulación sobre el mercado interno y alguna autonomía financiera y productiva de un estado inductor de la industrialización. Con esta intensidad y con estas particularidades propias, la mayoría de los países de América Latina van, ya en los años 90, a seguir el ejemplo de México, pionero en esta nueva aventura económica.

\section{LOS AÑOS 90: POLÍTICAS NEOLIBERALES Y REESTRUCTURACIÓN INDUSTRIAL}

El primer presidente electo directamente después de décadas de dictadura y de lenta transición democrática, Fernando Collor de Mello en los primeros meses de 1990 entierra el moribundo desarrollismo y crea las bases para la nueva era neoliberal que marca los años 90. El sonado fracaso de su principal objetivo de gobierno -el combate a la inflación- y su expulsión de la arena política debido al impeachment que abrevió su mandato no deben eludir el hecho de que él dio inicio a una serie de cambios que balizan la discusión sobre el desarrollo hasta hoy, por lo menos en dos sentidos más importantes de lo que se pretende discutir aquí: la redefinición del conflicto entre estabilización y desarrollo convirtiéndolo en el primer impasse de la economía y el completo cambio de sentido en la orientación general de la política industrial.

La prioridad absoluta concedida al combate a la inflación y el modo drástico -para no decir desgraciado- de su implantación ya en el inicio del gobierno sería el principal elemento para orientar las decisiones económicas: la profunda recesión de los años 90/92 y su corolario de desempleo y quiebra de empresas señalarían de forma duradera que la tradición brasileña de priorizar el crecimiento era un difunto a ser reverenciado nostálgicamente ${ }^{14}$.

La novedad no estaba en el hecho de la recesión en sí, sino en su perversa combinación con los condicionantes estructurales, es decir, la nueva política industrial del gobierno, que se pretende aquí sintetizar ${ }^{15}$. La estrategia consistía en una articulación

(12) Aquí el mejor ejemplo fueron las sanciones norteamericanas a un amplio conjunto de exportaciones brasileñas como contestación a la reserva del mercado informático y a la negación de las patentes de la industria farmacĚutica (ERBER y VERMLUM 1993:40/41).

(13) A este respecto ver FIORI (1995).

(14) Para un examen más detenido del contexto macroeconómico del gobierno Collor, ver ERBER Y VERMULM (1993:23/29) y el artículo de Bernardo Macedo en esta misma edición.

(15) Para un estudio más detallado sobre la política industrial en este periodo, ver BATISTA (1993:237)y ERBER y VERMULM (1993:41). 
entre iniciativas destinadas a presionar a los agentes privados para incrementar su cualificación técnica y económica (política de competición) e instrumentos de estímulo a la inversión y a la modernización (medidas de competitividad). A través de este movimiento de "pinza", se pretendía al mismo tiempo forzar a las empresas a reducir su distancia en relación a los patrones manejados internacionalmente y a crear condiciones que hiciesen esto posible.

La política de competitividad consistía básicamente en la triada neoliberal: privatizaciones, desregulación y una abrupta apertura comercial ${ }^{16}$. De los tres, este último elemento fue el más decisivo en la reorientación del sector privado: comenzando con el desmantelamiento amplio de las barreras tarifarias, y avanzando a través de un cronograma célebre de reducción de las tarifas de importación ${ }^{17}$, la apertura comercial introdujo una creciente presión de fabricantes extranjeros sobre la industria nacional que se convertiría rápidamente en el principal condicionante de las decisiones estratégicas de las empresas nacionales y multinacionales instaladas en Brasil. De forma complementaria fueron eliminadas varias restricciones a la entrada de empresas extranjeras, siendo el mejor ejemplo el fin de la reserva del mercado de la informática ${ }^{18}$.

En relación a las medidas de competitividad, la primera observación se refiere a su concepción: contrariando toda la tradición del ISI, las nueva políticas industriales eran de naturaleza horizontal, es decir, desprovistas de los elementos de targeting que permitían favorecer la adaptación de sectores específicos, o al menos reducir sus dificultades. O sea, más que una política industrial de desarrollo, volcada a la transformación estructural del aparato productivo, la estrategia gubernamental buscaba el fortalecimiento de los sectores industriales que ya disponían de ventajas comparativas, sea por la disponibilidad de recursos naturales, sea por la explotación de mano de obra barata.

Formada por algunos programas de incentivos a las inversiones en modernización técnica, una mejoría en la calidad de los productos y de la competitividad internacional, esta directriz de política incluía también estímulos a la ampliación de los gastos en investigación y desarrollo. Los resultados de estas medidas, necesariamente proyectados para el medio y largo plazo, dada su naturaleza, fueron sobremanera reducidos por el hecho de que el gobierno había puesto poco énfasis en su puesta en marcha: le faltaron recursos a un Estado que estaba siendo progresivamente desestructurado por la ofensiva neoliberal, en concreto, por la reforma administrativa (dimisiones, extinción de órganos, etc.) e indirectamente por la severa crisis fiscal provocada por los sucesivos y malogrados planes de estabilización.

Una evaluación de los resultados de esta estrategia de pinza debe comenzar por el reconocimiento, a menudo escamoteado por los críticos, de que ésta trajo innegables avances en el comportamiento empresarial: en varios de los principales segmentos industriales, hubo en los años 90 un esfuerzo de reestructuración del parque productivo y de las prácticas de gestión que no

(16) Además de la referencia de la nota anterior, ver también IESP (1993) para un balance de la polí tica de privatización del Gobierno Collor.

(17) La tarifa media, según el cronograma inicialmente definido, debía caer del $32,2 \%$ al $14,2 \%$ entre 1990 y 1994. En realidad el cronograma fue acelerado más adelante, y nuevas facilidades para la importación (por ejemplo, los bienes de capital) fueron creadas. Para un examen más detallado, ver ERBER Y VERMULM (1993:43/46).

(18) Cabe subrayar que en este momento la nueva geopolítica económica ya se convirtió en un elemento crucial: "la necesidad de renegociar la deuda externa según cánones del Consenso de Washington concedió prioridad máxima a las medidas de apertura, desregulación y privatización" (ERBER Y VERMULM 199:55) encontraron paralelo en ningún otro momento de la historia brasileña. En muchos otros sectores, sin embargo, puede observarse un downgrading industrial (es decir, un deterioro de la matriz productiva debido a la eliminación de actividades manufactureras y de proyectos más nobles) también sin precedentes. El mejor ejemplo es el complejo electrónico: pasó a importar muchos más productos acabados y componentes (sobre todo los más intensivos en tecnología) que antes, reduciendo o eliminando importantes segmentos de la cadena de valor agregado.

Incapaz de exponer una evaluación global de los resultados de esta política, incluso por falta de espacio, me limito a presentar el cuadro que es más común entre los analistas: en general, la industria brasileña está hoy mucho más capacitada para enfrentar la competición internacional que hace cinco o seis años atrás; cabe destacar que la reestructuración no fue homogénea, habiendo avanzado en varios sectores, mientras que en otros en realidad retrocedieron, sea porque se ha dejado de producir localmente y se importa (una especie de ISI al revés) sea por la eliminación de empresas. Lo importante es retener que los progresos registrados se deben casi exclusivamente a la política de competitividad, dado que los estímulos ofrecidos a los elementos "activos" de la nueva política industrial fueron muy débiles y sus resultados sólo pueden ser evaluados en el futuro, dada la naturaleza lenta de sus impactos.

\section{El otro lado de la globalización: el Mercosur}

Existe una segunda dimensión del proceso de apertura comercial que merece un tratamiento específico, aunque breve. Más allá de su dimensión multilateral, el ingreso de Brasil en la globalización financiera, productiva y comercial se estructura mediante la formación del bloque regional constituido por los países del Cono Sur: Brasil, Argentina, Uruguay y Paraguay. Habiendo tenido inicio en los años 80, es en la presente década cuando el Mercosur se afirma como la principal referencia para el comercio y las inversiones en toda la región, rápidamente unificada sobre la forma de una Unión Aduanera ${ }^{19}$.

El fuerte incremento en los flujos de comercio e inversión dentro de la región derivados del Mercosur es hoy uno de los factores más importantes para explicar el dinamismo de ciertos sectores industriales, como el automotriz y el agroindustrial, entre otros. Por otro lado, la integración regional implicó una reducción importante de la autonomía de los Estados-miembros en la conducción de la política económica, más concretamente en la determinación de las barreras comerciales y otras políticas industriales asociadas al comercio externo. Aunque la lista de exenciones a la Tarifa Externa Común sea todavía significativa, su programada extinción a fin de siglo apuntan, en el medio plazo, el completo fin de la autonomía nacional en estas cuestiones.

Más que esto, la propia relación de Brasil con sus socios comerciales, ahora al amparo de la Organización Mundial del Comercio (OMC), condiciona la continuidad de la apertura mul-

(19) Recién en mayo de 1996 es cuando el Mercosur cobra existencia jurídica definitiva, con la ratificación del protocolo de Ouro Preto. No obstante, su implantación económica se dio a lo largo de toda la década de los 90 y muestra señales inequívocas de que ya es la principal referencia económica en la región. Basta decir que mientras el intercambio global de Brasil aumentó en 50\% entre 1991 y 1994 (apertura mul-

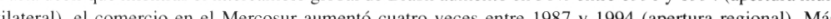
(jella e

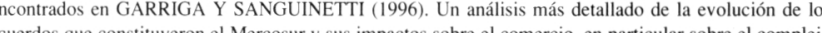
acuerdos que constituyeron el Mercosur y sus impactos sobre el comercio, en particular sobre el complejo automovilístico, están en COMIN (1996: cap.3 y sección 4.2). 
tilateral a la continuidad de los acuerdos establecidos por el Mercosur y sobre todo por la necesidad de homogeneizar, dentro del bloque, las condiciones de acceso a los mercados nacionales por parte de terceros países. En suma, la rápida apertura brasileña de los años noventa ya se presenta como un paso irreversible, dado que su poder de regulación sobre el mercado interno está en buena medida transferido hacia un nuevo tipo de soberanía económica, ahora de ámbito regional y mucho más limitado por las nuevas reglas de la OMC. El principal instrumento de inducción a la industrialización en la fase ISI, representado por este poder de regulación, vive tal vez sus últimos momentos.

\section{EL PLAN REAL Y LA SEGUNDA FASE DE LAS REFORMAS LIBERALIZANTES}

El Plan Real, implantado entre 1993 y 1994, es el gran punto de ruptura de la economía brasileña en esta década. A través de una ingeniosa reforma monetaria y algunas medidas complementarias de naturaleza fiscal, el entonces ministro de Hacienda y hoy presidente de la República, Fernando Henrique Cardoso, realizó la hazaña que se consideraba imposible dados los recurrentes fracasos anteriores: acabar con el descontrol inflacionario que hace más de diez años obstaculizaba la política económica y en diversas ocasiones amenazó destruir todo el tejido económico con el riesgo de hiperinflación. Al revertirse las expectativas más pesimistas del gobierno Collor y al ampliar el poder de consumo de la población de baja renta, la estabilización empujó una tendencia al crecimiento económico que ya se manifestaba antes de la reforma monetaria y generó un nuevo ambiente macroeconómico, todavía incierto, pero positivo.

Por otro lado, el gobierno Cardoso debe ser visto también como una continuación del gobierno Collor. Desde el punto de vista estratégico, es decir, de la base política que determina los principales objetivos y medios de la administración, lo que se percibe es un reciclaje de muchas fuerzas políticas perjudicadas por el impeachment, ahora con el ropaje de una coalición de centro derecha con discurso socialdemócrata. Es por ésto que se puede hablar de una segunda puesta en marcha del Consenso de Washington en Brasil, ahora con bases económicas y políticas más sólidas, ambas derivadas del Plan Real.

Por más que se busquen diferencias entre el anterior y el actual gobierno, el hecho es, desde el punto de vista práctico, que las orientaciones estratégicas son muy similares: apertura, privatizaciones y desregulación continúan siendo las directrices básicas tanto del cotidiano del actual gobierno como de sus propuestas de reformulación constitucional todavía en curso en el Poder Legislativo. Desde el punto de vista de la política industrial, esto significa una cuasi completa continuidad desde 1990. Poco se añadió en términos de competitividad, sin ampliar significativamente el ya limitado y contradictorio impacto sobre la modernización antes menciona$\mathrm{do}^{20}$. El incentivo a una creciente competición internacional permaneció siendo el motor principal de la reestructuración productiva, cuya trayectoria bifurcada -modernización y crecimiento en algunos sectores, downgradinge desindustrializador en otros- impide evaluaciones y previsiones genéricas sobre el futuro industrial.

(20) Más detalles en COMIN (1996:36).
Pero el Plan Real aumentó el último elemento que permaneció ausente -en una comparación con otros signatarios del Consenso de Washington en América Latina- en la primera fase neoliberal: la utilización del cambio como principal instrumento de contención de la subida de precios. En la implantación de la nueva moneda, la conversión de precios y el nuevo sistema de fijación de tasa de cambio (entre junio y septiembre de 1994) trajo consigo una desvalorización de ésta del orden del 15\%. No hay dudas de que esta estrategia fue el principal soporte de la trayectoria de baja inflación: de ahí que podamos hablar de un anclaje cambiario como núcleo del Plan Real ${ }^{21}$.

Esta estrategia de estabilización macroeconómica incluye además la política comercial: junto a esta maxivalorización de la moneda, nuevas reducciones tarifarias (dentro y fuera del Mercosur) abrieron camino para aquello que sería conocido como el "lupanar de las importaciones": el montante por este rubro pasa según los datos oficiales de 25.000 millones de dólares en 1993 a 33.000 millones en 1994, alcanzando los casi 26.000 millones en el primer semestre de 1995. De forma indirecta, la displicencia en la implantación de reservas comerciales -como instrumentos para combatir la concurrencia desleal (antidumping), comunes a todas las economías abiertas del mundo- dejó a la industria nacional excepcionalmente desprotegida frente a la competición internacional.

De este modo, el ministro de Hacienda, Fernando Henrique Cardoso, consiguió el milagro económico que garantizaba su elección como presidente a fin de 1994: crecimiento económico, reducción vertiginosa de la inflación y alguna redistribución de la renta a favor de los sectores populares. La evidente inconsistencia macroeconómica de este ingenioso "arreglo" de medidas económicas no tardó en exigir un cambio por parte del gobierno. Desde el inicio de su mandato, en enero de 1995, quedaba claro para Fernando Henrique Cardoso que el mantenimiento de la estabilidad, su principal compromiso político, solamente podría ser sostenido a expensas de la performance de otras variables económicas. Presionado por la crisis mexicana de 1994, y registrando déficits asustantes en la balanza de $\operatorname{pagos}^{22}$, no había otra alternativa para la política económica del país que la reversión de la trayectoria de crecimiento ${ }^{23}$. Esto fue logrado básicamente mediante una brutal elevación de la tasa de interés interna, acompañada de medidas discrecionales de contención del crédito al consumo. Los cambios sirvieron de forma doble a los objetivos de la estabilización: por un lado, la remuneración estratosférica de los capitales especulativos compensa los temores causados por el desastre mexicano; por otro, contienen la expansión de la demanda interna, desanimando la afluencia de importaciones.

21) Para un análisis detallado de la estrategia de estabilización del Plan Real y una comparación con Argentina y México, ver BATISTA JR. (1996). El autor añade además que la valorización cambiaría continuó creciendo en la coyuntura electoral de fin de 1994, alcanzando cerca del $30 \%$, permaneciendo estable desde entonces. Ver también el referido artículo de Bernardo Macedo.

(22) "El déficit por cuenta corriente en el primer semestre de 1995 correspondió al 53\% de las exportaciones de mercancías y al $4 \%$ del PIB (...) El desequilibrio externo producido en esa primera fase del Plan Real también se aproximaba peligrosamente a los niveles observados en los años que antecedieron a la criReal también se aproximaba peligrosamente a los niveles observados
sis de la deuda externa en los años 80", en BATISTA JR. (1996:22).

(23) Los datos agregados indican que todavía se mantiene algún crecimiento económico, proyectado entre el 2 y el 2,5\% para este año. La industria, sin embargo, da claras señales de pérdida de dinamismo, configurando en la mejor de la hipótesis, una estancamiento en la producción y en las ventas. Bastan algunas cifras: desde mayo de 1995, cuando aparecen los efectos de la segunda fase del Plan Real, el indicador de las variaciones de la producción industrial fue positivo sólo en dos meses (datos oficiales del IBGE), haciendo que la acumulación anual hasta marzo de 1996 fuese del orden de -3.9\%: según la Confederación Nacional de Industria. el nivel de empleo industrial cayó durante el $11^{\circ}$ mes consecutivo en marzo de 1996. 
Esta breve exposición de la actual situación brasileña nos remite al núcleo principal de esta discusión, porque el gobierno Cardoso nace enfrentando el que hoy parece ser el gran dilema del desarrollo brasileño, y por extensión, de otros países en situación similar: estabilización y crecimiento, en el nuevo contexto global, son metas que muy difícilmente pueden ser compatibilizadas. Dicho de otro modo, la estabilización puede ser precondición para el desarrollo, pero la manera cómo es alcanzada y cómo es mantenida, en el caso brasileño, inviabilizan cualquier crecimiento económico que por lo menos sirva para acompañar las ya por sí mediocres tasas de evolución de la producción y el comercio mundiales.

En un contexto de cuasi absoluta pérdida de soberanía sobre el mercado interno, y apostando por una incierta y abrupta integración comercial y financiera con el resto del mundo, los márgenes de maniobra de la política económica son hoy más estrechos que nunca. En ausencia de políticas industriales de corte vertical y que dispongan de alguna autonomía frente a los dictámenes de las políticas de estabilización, el desarrollo futuro de la industria en Brasil deberá recorrer caminos muy difíciles. Dada la coyuntura de estagnación o de bajo crecimiento que se puede esperar de la actual configuración macroeconómica, sin ningún estímulo activo por parte del gobierno, el futuro de la industria es incierto.

Dicho de otro modo, la creciente dependencia externa gestada en el final del periodo ISI, debido sobre todo al endeudamiento externo, parece haber anulado completamente aquello que había sido hasta aquel momento su principal éxito, ampliar la autonomía nacional en un contexto internacional hostil que, hoy día, tiene por característica básica la ampliación de la brecha entre países ricos y pobres. La estrategia neoliberal que suplanta la ISI no sólo no es capaz, por su propia naturaleza antiintervencionista, de revertir los daños de esta situación heredada sino que además empuja al país a una nueva cota de fragilización productiva y financiera frente a los países centrales del capitalismo. El dilema anterior se convierte entonces en la siguiente paradoja: es preciso estabilizar y conceder ventajas a los capitales internacionales (tarifas, patentes, privatizaciones, desregulaciones, etc.). para que ellos ingresen en la economía interna, dinamizándola con sus recursos y técnicas tan necesarias; sin embargo, para hacer esto, es preciso disciplinar a los agentes económicos internos -empresarios y trabajadores- para que acepten las condiciones impuestas por la competición internacional, lo que implica limitar el crecimiento de la renta interna y eliminar justamente el principal atractivo para los capitales foráneos, a saber, el gran y dinámico mercado interno.

\section{UN CONTRAPUNTO: LA INDUSTRIA AUTOMOVILÍSTICA EN BRASIL}

Obviamente, no se pretende aquí presentar una solución para los problemas y preocupaciones levantadas en la sección anterior. Además, no parece haber demasiada claridad por parte de nadie en cuanto a estas cuestiones, dada la novedad de los desafíos. Dentro del propio gobierno es visible la existencia de profundas divergencias en cuanto al diagnóstico y a las estrategias de desarrollo que deben ser adoptadas ${ }^{24}$. Entre otras cuestiones

(24) Llamé la atención sobre este problema en COMIN (1996:37). Este apartado está basado princ palmente en ese trabajo. importantes, estas divergencias afectan primordialmente a la utilización de la regulación del mercado interno como un mecanismo de atracción de inversiones o de consolidación del parque productivo o, al contrario, el uso de la apertura comercial para contener el aumento de precios internos. En el caso del sector automotriz, la primera orientación pasó recientemente a prevalecer sobre la segunda. Esta sección pretende discutir rápidamente la nueva política industrial para el complejo automovilístico, mostrando cómo una orientación no tan neoliberal levanta perspectivas mucho más optimistas que las analizadas anteriormente.

En los últimos años, América Latina en general y Brasil en particular, se convirtieron en una de las áreas de mayor demanda de vehículos automotores de todos los tipos. La integración creciente entre mercados y parques productivos de Brasil y Argentina, a través del Mercosur, conllevó un impulso adicional importante a las inversiones de las grandes firmas, incluso, algunas que acaban de entrar en estos países. El crecimiento ininterrumpido de ventas y producción interna, así como la ampliación sin precedentes de las inversiones en todo el complejo presentan una trayectoria bastante dinámica para el sector en Brasil y en el Mercosur.

Pero este dinamismo no se explica por los mejores condicionantes del mercado, aunque ellos sean cruciales: la orientación de la política industrial fue y continúa siendo decisiva. La simple apertura comercial en un momento de crecimiento de la demanda interna, como ocurrió en la primera fase del Plan Real, trajo consigo el aumento explosivo de la importación, lo que tuvo como consecuencia una elevación sin precedentes de la pérdida de divisas por la adquisición de vehículos en el exterior ${ }^{25}$.

Contando con un Régimen Automotriz desde 1991, Argentina ha sido capaz de atraer crecientes inversiones de las firmas automotrices, en detrimento inclusive de inversiones en Brasil ${ }^{26}$. Al igual que allí, desde el final del gobierno Collor, Brasil también viene dispensando un tratamiento privilegiado al sector automovilístico. Mientras que otros ramos de la industria son objeto de la política industrial sólo en su dimensión pasiva, es decir, en las políticas de competición, la industria automotriz cuenta con una serie de incentivos fiscales y tarifarios que han viabilizado su trayectoria reciente, dinámica y que muestra perspectivas prometedoras para los próximos años. Sin espacio para analizar detenidamente este proceso, menciono apenas el elemento central del nuevo Régimen Automotriz, que comenzó a ser implantado a mediados de 1995 y que en este momento ya presenta señales claras de éxito ${ }^{27}$.
(25) La tabla 10 en COMIN (1996:60) muestra esto claramente: la adquisición de casi 200.000 vehículos en 1994 (más de la demanda acumulada entre 1990/1993 cuando comienza a funcionar el nuevo régimen de apertura comercial de Collor) y la entrada de 400.000 unidades más en el año siguiente son ilustrativas de la magnitud del "lupanar de las importaciones". Las proyecciones realizadas para el año 1995, antes de las medidas anticonsumo mencionadas y de la nueva política industrial discutida a continuación, señalaban la posibilidad de que el país gastase más de 10 mil millones de dólares con estas importaciones, un montante muy superior a la capacidad importadora del país en las actuales condiciones de restricción externa.

(26) Con la nueva política, la transferencia de inversiones parece haber cesado. Destáquese que lá posibilidad de nuevas medidas en Brasil están dadas por la existencia de condiciones similares en Argentina. Al menos fue este el argumento utilizado por el país para justificar las nuevas restricciones al comercio en el ámbito de la OCM. Esto muestra cuánto de la soberanía nacional está ya comprometida en la regulación del mercado dada la nueva inserción internacional.

(27) Este análisis fue efectuado en COMIN (1996:cap.1). Falta espacio también para discutir la mportante dimensión política que este proceso presentó en su inicio: contrariando la orientación extremadamente impositiva que toda la política económica presentó en los años 90 , los cambios en la política industrial para el sector automotriz fueron realizadas, entre 1992 y 1993 en el ámbito de una Cámara Sec torial que reunía empresarios, trabajadores y gobierno en todas las áreas del complejo y que representó un importante avance democrático en la elaboración de la política económica en el Brasil. Infelizmente. el nuevo gobierno, sobre todo después de la toma de posesión del nuevo presidente, ha revertido esta tendenci y la nueva polítca económica vuelve a los tradicionales moldes de la historia brasileña por medio de yla Sobre ese a Sobre este asunto, ver CARDOSO Y COMIN (1995) 
Después de las sucesivas subidas de la tarifa arancelaria para vehículos, hoy en el 70\%, el mayor porcentaje desde la apertura en 1990, el nivel de protección, a pesar de la valorización cambiaria, se volvió suficiente para estimular la producción interna: dentro de otras medidas de política industrial, el gobierno estableció una exención de 50\% sobre la referida tarifa para vehículos importados por las empresas con fábricas en Brasil, exigiendo como contrapartida, compromisos explícitos de inversión y exportaciones. Todas estas firmas automotrices y algunos fabricantes de componentes ya se comprometieron a través del nuevo programa, a ampliar la producción y a elevar sustancialmente las exportaciones de componentes y vehículos acabados. Además, el nuevo régimen ha sido eficaz en atraer también las inversiones de nuevas firmas (al menos seis de las más importantes de Europa, Japón y Corea) al país. Globalmente se proyectan inversiones del orden de los 17.000 millones de dólares hasta el final de la década, plazo previsto para el fin del programa, cifra sin precedentes en toda la historia de la industria.

En suma, contrariando la orientación horizontal de la política industrial, el gobierno estableció para el sector automovilístico una estrategia de corte claramente vertical: targeting preciso en el complejo, metas pactadas de producción, inversiones y exportaciones, con plazos definidos, así como los recursos e instrumentos de regulación claramente establecidos. Cabe resaltar que, aunque haya tenido como punto de partida la regulación de los mercados internos, ahora debidamente protegidos, la nueva política no implica un retorno a los viejos patrones de la ISI: ahora el mercado interno y externo no se oponen, al contrario, se complementan en una estrategia que procura inducir el crecimiento para adentro al mismo tiempo que crea las condiciones para una inserción externa competitiva y que admite una creciente afluencia de importaciones, compatibilizando de este modo globalización con desarrollo interno ${ }^{28}$.

\section{CONSIDERACIONES FINALES}

Este artículo procuró poner en evidencia los impasses y dudas que acechan a la continuidad del desarrollo en Brasil más que proponer soluciones o sugestiones. La verdad es que no existe hoy una comprensión clara sobre lo que podría ser una estrategia de desarrollo que fuese capaz de, al mismo tiempo, superar las deficiencias del modelo ISI en el nuevo contexto de globalización económica y asegurar un mínimo de crecimiento y prosperidad para los países de industrialización tardía. Nos falta un cuerpo teórico que, a ejemplo del elaborado por la Comisión Económica para América Latina (CEPAL/ONU), permita, ahora sobre nuevas bases, una nueva ola de desarrollo, como aquella que caracterizó a muchos países de América Latina entre los años 30 y 70 .

A pesar de este cuadro general de incertidumbres, esperamos que este artículo haya sido capaz de poner de manifiesto dos aspectos cruciales de la actual situación brasileña, en alguna

(28) Hay aquí una cierta dosis de optimismo: en este exacto momento (mayo de 1996), Brasil se prepara para defender en la OCM la nueva política industrial para el sector. Amenazada por la fuerte oposición de los EE.UU y Japón en esta organización, el conflicto evidencia la nueva postura del capitalismo ción de los EE.UU y Japón en esta organización, el conflicto evidencia la nueva postura del capitalismo
central, a saber, obstaculizar los esfuerzos de los países atrasados en buscar autonomía para su hacer su procentral, a saber, obstaculizar los esfuerzos de los países atrasados en buscar autonomía para su hacer su pro-
pio camino industrial, como intento de reducir su atraso. La diferencia con los tiempos de la ISI, cuando todavía era posible explotar las divergencias diplomáticas y los conflictos económicos entre las potencias capitalistas, es bastante clara. medida generalizables a otros países. En primer lugar, está claro que el modelo neoliberal, aquí implantado sobre la égida del Consenso de Washington, sólo garantiza una nueva mayor dependencia externa y, como muestra incluso la experiencia de los países centrales, una mayor exclusión social. Nada indica que, como profesan los defensores del modelo, una vez superadas las dificultades iniciales, inherentes a la conversión a las nuevas reglas, las fuerzas del mercado irán progresivamente a ejecutar la tan deseada integración competitiva en el mercado mundial, generando empleos y renta para la economía nacional. Si no es cierto, sí es probable que la apertura irrestricta aumente la dependencia, trayendo el riesgo inclusive de una reversión industrial, aproximando nuestras economías a la fragilidad externa que caracterizó a América Latina antes de la ISI.

En segundo lugar, continúan presentes las oportunidades embutidas en la condición de atraso, es decir, el enorme potencial económico representado por amplios mercados domésticos todavía a ser integralmente explotados (y ahora dinamizados por las perspectivas de integración regional). Como demuestra el caso de la industria automovilística, la utilización consciente y planificada de la regulación al mercado interno es todavía un mecanismo eficiente para modelar las desventajas impuestas por las condiciones de mercado con el objetivo de reforzar el parque industrial interno y garantizar una mejor inserción en el nuevo contexto global.

\section{REFERENCIAS BIBLIOGRÁFICAS}

BAER, Werner (1985): A Industrialização e o Desenvolvimento Econômico do Brasil. 6ª ed. Rio de Janeiro, Editora da Fundação Getulio Vargas, 593 pp.

BAPTISTA, Margarita Afonso Costa (1993): "Política Industrial e Desestruturação Produtiva", In DESEP, Crise Brasileira (Anos oitenta e governo Collor). São Paulo, Desep/CUT e CGIL, 361 pp., 1993, pp. $219 / 274$.

Batista JR., Paulo Nogueira (1996): Plano Real: Estabilização Monetária e Desequilíbrio Externo. Texto para Discussão (50), São Paulo, EAESP/FGV, 39 pp.

Cardoso, Adalberto Moreira \& Comín, Álvaro Augusto (1995): "Câmaras setoriais, modernização produtiva e democratização nas relações de trabalho no Brasil (A experiência do setor automobilístico)". In Villas Boas, Glaudia \& Gonçalves, Marco Antonio (Org.), O Brasil na Virada do Século (O debate dos cientistas sociais). Rio de Janeiro, Relume Dumará, 299 pp., 1995, pp. 35/52.

Cомín, Alexandre (1996): Globalização e Relações de Trabalho na Indústria Automobilística Brasileira. Relatório Final do Projeto Comparativo Internacional Brasil-México-Colômbia, São Paulo, Convênio Universidade de Bremem-Cebrap, 101 pp.

ERBer, Fábio \& Vermulm, Roberto (1993): Ajuste Estrutural e Estratégias Empresariais. Rio de Janeiro, IPEA, 271 pp.

Evans, Peter (1984): Class, State, and Dependence in East Asia: Lessons for Latin Americanists. Mimeo, Brown University, 37 pp.

FAJNZYlBer, Fernando. (1983): La Industrialización Trunca de América Latina. México, D.F., Editorial Nueva Imagen, 416 pp.

FIORI, José Luis (1986): A Crise do Estado Desenvolvimentista no Brasil (Uma hipótese preliminar). Textos para Discussão (88), Rio de Janeiro, IEI/UFRJ, $111 \mathrm{pp.}$

_ (1995): O Vôo da Coruja (Uma leitura não liberal da crise do Estado desenvolvimentista). Rio de Janeiro, Ed UERJ, 133 pp. 
FurTado, Celso (1982): Formação Econômica do Brasil. 18 ed. São Paulo, Companhia Editora Nacional, 248 pp.

Garriga, Marcelo \& SAnguinetti, Pablo (1996): Coordinación Macroeconómica en el Mercosur (Ventajas, desventajas y la práctica). Serie Prosur, Buenos Aires, Fundación Friedrich Ebert, 19 pp. IESP (1993): Processo de Privatização no Brasil (A experiência dos anos 1990-92). São Paulo, IESP/Fundap, 338 pp.

PINHEIRO, Armando Castelar (1987): Estratégias de Desenvolvimento (America Latina vs. Leste Asiático). Textos para Discussão Interna (128), Brasília, IPEA.
SHAPIRO, Helen (1994): Engines of Growth (The state and transnational auto companies in Brazil). Cambridge, University of Cambridge, $267 \mathrm{pp}$.

SuzIGAN, Wilson (1996): "Experiência histórica de política industrial no Brasil”, en Revista de Economia Política, São Paulo, Centro de Economia Política, 16 (61), pp. 5/21.

TAVARES, María da Conceição (1972): Da Substituição de Importações ao Capitalismo Financeiro (Ensaios sobre economia brasileira). $11^{\underline{a}}$ ed. Rio de Janeiro, Zahar Editores, 263 pp.

\section{RESUMEN}

Empezando por un análisis del patrón de industrialización brasileño y las causas de su fracaso a partir de los 80 en un contexto de ideología neoliberal, el autor analiza los nuevos parámetros de la política industrial de los años 90 . A la luz del nuevo contexto de estabilización y de integración regional, se observan los diferentes impactos que esta estrategia tiene sobre la estructura productiva.

\section{ABSTRACT}

Begining with an analysis of the brazilean industrialization pattern and the causes of its failure form the eighties in a context of neoliberal ideology, the author analyzes the new parameters of the industrial policies in the nineties. In this new context of stabilization and regional integration, it is observed the different impacts thar this strategy has over the productive structure.

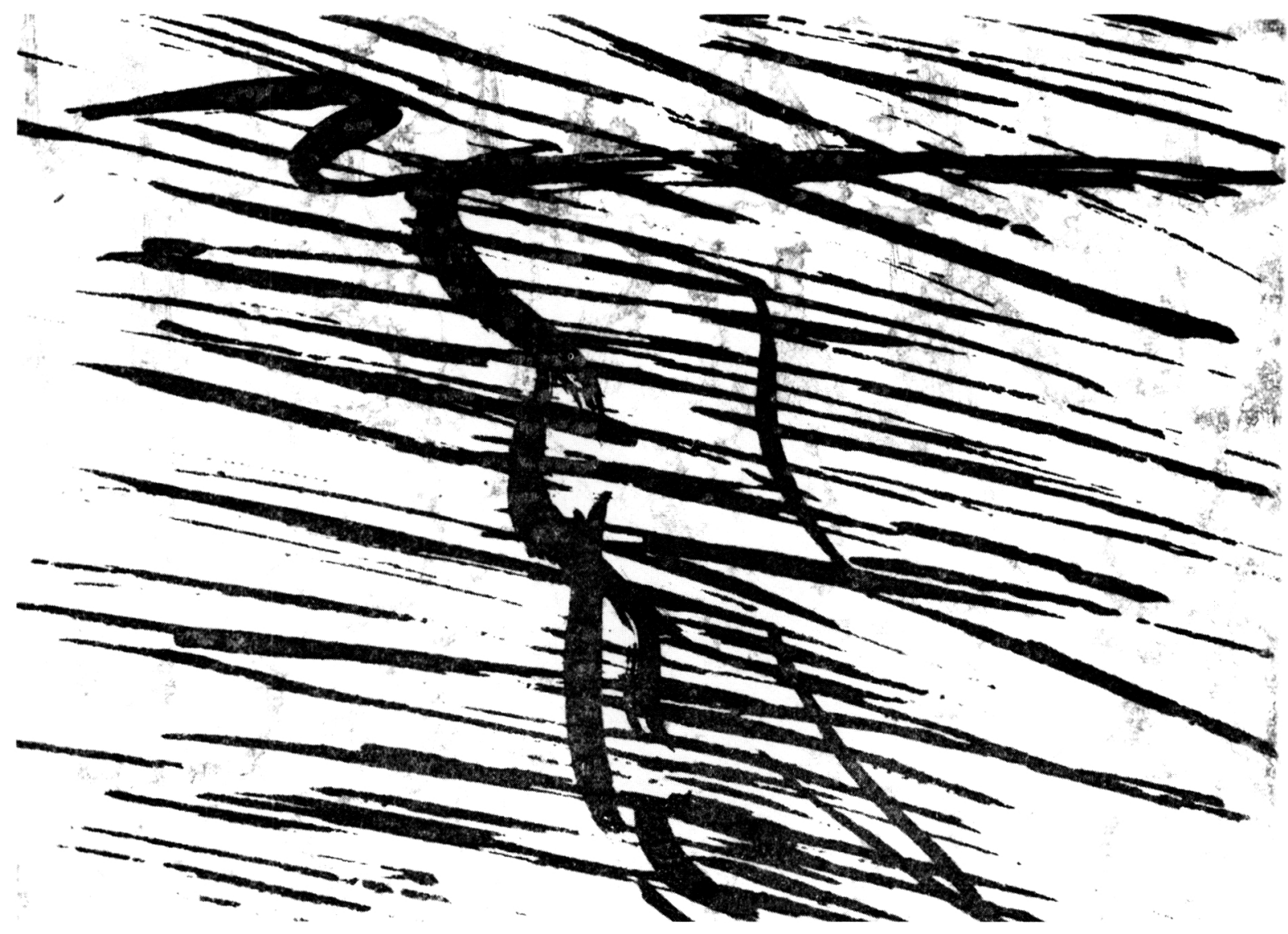

